\title{
Rashba spin-orbit coupling and spin relaxation in silicon quantum wells
}

\author{
Charles Tahan and Robert Joynt \\ Physics Department, University of Wisconsin-Madison
}

(Dated: January 2004)

\begin{abstract}
Silicon is a leading candidate material for spin-based devices, and two-dimensional electron gases (2DEGs) formed in silicon heterostructures have been proposed for both spin transport and quantum dot quantum computing applications. The key parameter for these applications is the spin relaxation time. Here we apply the theory of D'yakonov and Perel' (DP) to calculate the electron spin resonance linewidth of a silicon 2DEG due to structural inversion asymmetry for arbitrary static magnetic field direction at low temperatures. We estimate the Rashba spin-orbit coupling coefficient in silicon quantum wells and find the $T_{1}$ and $T_{2}$ times of the spins from this mechanism as a function of momentum scattering time, magnetic field, and device-specific parameters. We obtain agreement with existing data for the angular dependence of the relaxation times and show that the magnitudes are consistent with the DP mechanism. We suggest how to increase the relaxation times by appropriate device design.
\end{abstract}

\section{INTRODUCTION}

Electron spins in silicon have been proposed as an attractive architecture for spintronics and quantum information devices. The inherently low and tunable spin-orbit coupling (SOC) in silicon heterostructures and the possibility of eliminating hyperfine couplings by isotopic purification bodes well for quantum coherent spin-based qubits and spin transport. Early experiments together with theory have shown that coherence times can be upwards of three orders of magnitude longer than in GaAs. 1, 2, 3, 4]

Energy relaxation of localized spin states has attracted theoretical attention [5, 6, 7, 8] and experimental effort [3, 9] for decades, and this activity has recently revived in the context of quantum computation. The idea is to store quantum information in the spin of a single electron confined in a semiconductor structure, either attached to a donor atom or confined electrostatically in a quantum dot. Spin transport, also of great interest, encodes information in the spin states of an ensemble of electrons. In both cases, electron spin resonance (ESR) measurements of spin relaxation provide a key and available measure of spin coherence properties of electrons in silicon quantum wells, though not a one-to-one correspondence. Our aim in this paper is to explain some existing ESR results for silicon 2DEGs at low temperatures and to make predictions for future experiments.

The structures that concern us here are layered semiconductor devices of $\mathrm{Si}$ and SiGe. The active layer is the quantum well $(\mathrm{QW})$ that confines the electrons in the growth direction. This layer will be assumed to be composed of pure, [001] strained silicon. We shall also neglect any roughness or miscut at the the $\mathrm{Si} / \mathrm{SiGe}$ interfaces. Devices made in this way are commonly referred to in the semiconductor industry as MODFETs and are designed to maximize mobility. Figure 1introduces two example structures.

Extensive theoretical work has been done on spin relaxation in GaAs and other III-V materials. The developments that began with the theory of D'yakonov and Perel' 10, 11, 12] are most relevant for our purposes. These authors found that fluctuating effective magnetic fields due to momentum scattering in the presence of SOC is the dominant spin relaxation mechanism in semiconductor 2DEGs for low temperatures. Here, we start with this assumption and use a general spin-density matrix approach to calculate the relaxation times of a 2DEG in the presence of a static magnetic field, including explicitly the angular dependence. Understanding the angular dependence of the linewidth is important for comparison with ESR experiments and the extraction of physically relevant parameters such as the momentum relaxation time. We also calculate separately in a $k \cdot p$ formalism the Rashba spin orbit coupling parameter in silicon quantum wells. This is a key parameter in our calculation as well as for other spin control considerations, both negative and positive.

In the next section we discuss the origin and magnitude of the SOC in realistic heterostructures. The section following that presents our calculation. Lastly, we compare with experiment and discuss the implications for device design.

\section{SPIN-ORBIT COUPLING}

The strong macroscopic electric fields inside heterostructure QWs are important for understanding SOC, especially in silicon. These fields are also device-specific, so we carry out our calculations on the two representative structures in Figure 1 Both devices have square QWs, with equal barriers on the top and bottom interface. The first is typical 


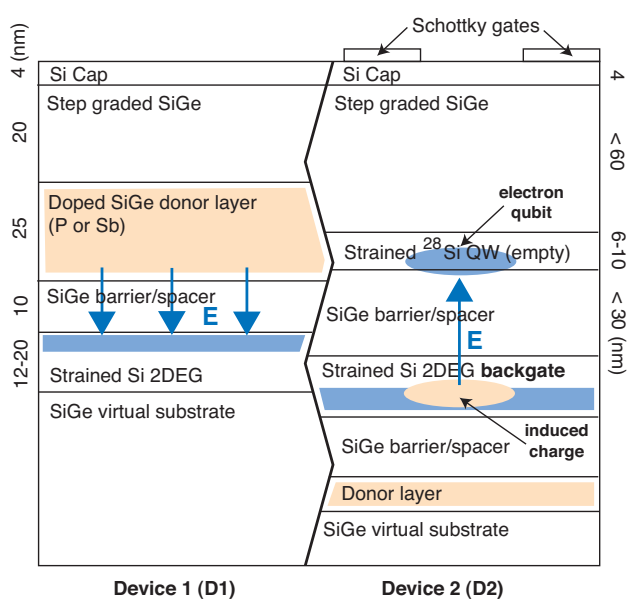

Figure 1: Strong, internal electric fields are common in silicon quantum well devices. D1: A typical, high-mobiliy SiGe heterostructure uses a donor layer to populate a high-density 2DEG. The charge separation results in an $E_{z} \sim 10^{6} \mathrm{~V} / \mathrm{m}$. D2: A proposed quantum dot quantum computer [13] which utilizes a tunnel-coupled backgate to populate the quantum well without the need for a nearby donor layer. Here, $E_{z}>10^{5} \mathrm{~V} / \mathrm{m}$ due to the image potential formed on the backgate.

of MODFETs and employs a donor layer above the QW in order to populate it. This charge separation produces an electric field between the two layers (across the barrier or spacer layer) which can be approximated by

$$
E_{z} \approx \frac{e n_{s}}{\epsilon_{0} \epsilon_{S i}}=-6 \times 10^{6} \mathrm{~V} / \mathrm{m}
$$

where $n_{s}=4 \times 10^{15} \mathrm{~m}^{-2}$ is the density of electrons in the 2DEG for Device 1, $e$ is the charge of an electron, and $\epsilon_{i}$ are the dielectric constants. We assume that the $Q W$ is populated only by donor-layer electrons, leaving an equal amount of positive charge behind. The second structure is one that has been proposed for use in a quantum computer device. 13] It utilizes a near-lying, tunnel-coupled backgate 2DEG ( $<30 \mathrm{~nm}$ away) together with Schottky top-gates to populate the QW selectively. This situation also results in a strong electric field due to the image potential on the back gate. For one qubit, this can be estimated as

$$
E_{z} \approx \frac{e}{4 \pi \epsilon_{0} \epsilon_{S i} d^{2}}=3 \times 10^{5} \mathrm{~V} / \mathrm{m}
$$

where $d=20 \mathrm{~nm}$ is the distance from the QW to the back gate for Device 2. Schottky top-gates and other device parameters can augment or reduce this growth-direction electric field nominally up to the breakdown field of silicon, $3 \times 10^{7} \mathrm{~V} / \mathrm{m}$, or the ionization energy of the electron. 14] Indeed, this field can actually be smaller than that due to the top-gates in certain dot configurations.

The shift of the electron $\mathrm{g}$-factor from its free-electron value $g_{0}=2.00232$ is one measure of SOC in a system. It is quite small in bulk silicon and depends on the magnetic field direction in the (elliptical) conduction band minima $\left(\Delta g_{\|} \approx-0.003, \Delta g_{\perp} \approx-0.004\right)$. [9] However, it is difficult to reliably extract the SOC strength in a 2DEG from $\Delta g$. Many parameters (e.g., strain, barrier penetration, Ge content $\left(g_{G e}=1.4\right)$, non-parabolicity of the band minima) influence the magnitude and sign of $\Delta g$ and it may show considerable sample dependence. The non-parabolicity effects are especially sensitive to the electron density within the QW and can hide the magnitude of SOC within a system. 15.

In these silicon heterostructures, SOC is dominated by inversion asymmetry within the device. The spin-orbit (SO) Hamiltonian to first order in momentum is given in an arbitrary electrostatic potential $V$ by

$$
H_{S O}=\frac{\hbar}{4 m^{2} c^{2}} E_{z} \vec{\sigma} \cdot(\hat{z} \times \mathbf{p}),
$$

where $\sigma_{i}$ are the Pauli matrices. Note that the effective magnetic field that acts on the spin is in the plane of the layer. In Si heterostructures, the macroscopic fields, which do not average out, are more important than the atomic electric fields. In the noncentrosymmetric III-V materials such as GaAs, this is not necessarily the case and the resulting Dresselhaus or bulk inversion asymmetry fields are usually dominant. The asymmetry considered here, due either to an interface, charge distribution, or external potential, is usually called Rashba or structural inversion asymmetry. 
The Rashba term comes directly from the SO Hamiltonian if we assume one, dominant symmetry-breaking electric field in the structure and average over a momentum state. In a QW, as we have pointed out above, the electric field is in the growth $(z)$ direction and thus the $z$-component of the above dot-product is selected and we obtain

$$
H_{R}^{2 D}=\alpha\left(p_{x} \sigma_{y}-p_{y} \sigma_{x}\right) \propto E_{z}(\vec{\sigma} \times \mathbf{p})_{z}
$$

which is then the Rashba-Bychkov Hamiltonian. [16]

Strictly speaking, as de Andrade e Silva et. al. point out [17], the conduction-band-edge profile, $E_{c}$, and the space charge separation (or applied electrostatic field), $E_{z}$, contribute separately and sometimes dissimilarly to the SOC. For example, the wavefunction discontinuity (band offset) across a material-interface can cause Rashba spin-splitting itself. However, in devices of the type considered here, the macroscopic field should be the main contribution. These same authors have derived an expression for $\alpha$ in the Kane model for GaAs. We have adapted their work for Si, using a 5-parameter 8-band Kane model. This is 8 bands including spin, which means just the lowest conduction band and the three highest valence bands. By calculating the breaking of the degeneracy between the spin-up and spin-down states of the lowest conduction band, we find

$$
\alpha=\frac{2 P P_{z} \Delta_{d}}{\sqrt{2} \hbar E_{v 1} E_{v 2}}\left(\frac{1}{E_{v 1}}+\frac{1}{E_{v 2}}\right) e\left\langle E_{z}\right\rangle,
$$

where we have taken the average of the electric field in the $z$-direction. Here $P=\hbar\left\langle X\left|p_{x}\right| S\right\rangle / i m, P_{z}=\hbar\left\langle Z\left|p_{z}\right| S\right\rangle / i m$, $\Delta_{d}=0.044 \mathrm{eV}$ is the spin-orbit splitting of the two highest conduction bands, $E_{v 1}=3.1 \mathrm{eV}$ is the direct gap of the strained sample, and $E_{v 2}=7 \mathrm{eV}$ is the gap between the conduction band minimum and the lowest of the three valence bands. (These are the 5 parameters mentioned above.) $m$ is the bare electron mass. The matrix elements that define $P$ and $P_{z}$ are to be taken between the cell-periodic functions of the indicated symmetry at the position of the conduction-band minimum. Unfortunately, these are not well known in Si, since other bands contribute. We may note that $P$ and $P_{z}$ are examples of momentum matrix elements that don't vary too much in III-V materials and Ge, [18] and are given approximately by $2 m P_{(z)}^{2} / \hbar^{2} \approx 22 \mathrm{eV}$. With these values we find that for devices of type 1 ,

$$
\alpha \approx 1.66 \times 10^{-6}\left\langle E_{z}\right\rangle \mathrm{m} / \mathrm{s}
$$

Previous Kane models for GaAs involve matrix elements at $k=0$. Our theory is new since it takes into account the proper symmetry of silicon with it's minima well away from the zone center.

Wilamowski et. al. [19], using conduction electron spin resonance (CESR), have measured $\alpha \approx 5.94 \mathrm{~m} / \mathrm{s}(\alpha e / \hbar=$ $0.55 / \sqrt{2} \times 10^{-12} \mathrm{eV} \cdot \mathrm{cm}$ in their units) 33] in a $\mathrm{Si}_{0.75} \mathrm{Ge}_{0.25} / \mathrm{Si} / \mathrm{Si}_{0.75} \mathrm{Ge}_{0.25} \mathrm{QW}$ where the strained-silicon layer was roughly $12-20 \mathrm{~nm}$. Carrier concentrations were $n_{s} \approx 4 \times 10^{15} \mathrm{~m}^{-2}$. These numbers correspond to our Device 1 parameters. Our equations then give, using Eq. 5]

$$
\alpha^{D 1} \approx 5.1 \mathrm{~m} / \mathrm{s} \text { for }\left\langle E_{z}\right\rangle=3 \times 10^{6} \mathrm{~V} / \mathrm{m} .
$$

Theory compares in order of magnitude and we believe that our estimation has some utility as a guide for device design.

For Device $2, \alpha^{D 2} \approx 0.25 \mathrm{~m} / \mathrm{s}$ for $\left\langle E_{z}\right\rangle=1.5 \times 10^{5} \mathrm{~V} / \mathrm{m}$. This device remains to be built. For Device 1 , we can also predict the zero magnetic field spin-splitting in a silicon 2 DEG using $\left|\epsilon_{+}-\epsilon_{-}\right| \leq 2 \alpha p_{F}$,

$$
2 \alpha p_{F}=2 \alpha \hbar \sqrt{\frac{4 \pi n_{s}}{4}} \approx 0.75 \mu \mathrm{eV}
$$

where 4 is the degeneracy factor in silicon (spin+valley). This has not yet been directly measured to our knowledge. Taking a Zeeman splitting of $g \mu_{B} B=0.75 \mu \mathrm{eV}$ with a g-factor of 2 , this implies an internal, in-plane, effective magnetic field - the so-called Rashba field - of roughly 62 Gauss, which is the direct result of SOC in the silicon 2DEG of Device 1.

Let's consider the relevance of our silicon SOC results to QC and spintronics. We note that the magnitude of the Rashba coefficient is much smaller than for GaAs. For the same electric field and 2DEG density as Device 1, a similar $\vec{k} \cdot \vec{p}$ theory for GaAs arrives at $\alpha_{G a A s} \approx 230 \mathrm{~m} / \mathrm{s}$. [17] But GaAs itself is not a high Rashba III-V semiconductor and is thought to be be dominated by Dresselhaus SOC $\left(\beta_{G a A s} \approx 1000 \mathrm{~m} / \mathrm{s}\right)$. [20] InAs-based heterojuctions, for example, may have orders of magnitude higher Rashba values. 21] This means that SOC effects in silicon devices will be much smaller, including decoherence and gating errors that are SOC-based. 

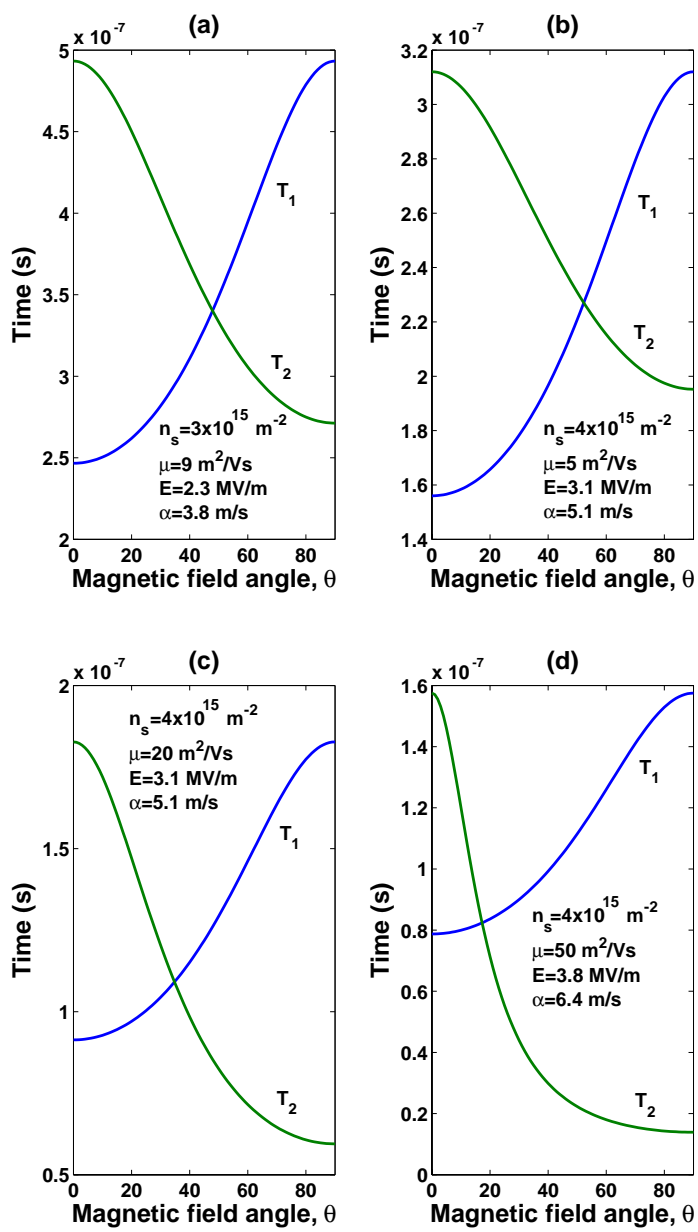

Figure 2: ESR linewidths in Gauss as a function of static magnetic field direction (where $\theta=0$ is perpendicular to the 2DEG plane) for specific values of 2DEG density and Rashba asymmetry. The quantum well is assumed to be completely donor-layer populated and as such, $\alpha$ is calculated directly with Eq. [5 as a function of the 2DEG density.

\section{SPIN RELAXATION}

We wish to consider the combined effects of the SO Hamiltonian

$$
H_{R}^{2 D}=\alpha\left(p_{x} \sigma_{y}-p_{y} \sigma_{x}\right)
$$

and the scattering Hamiltonian. The scattering may be from phonons or from static disorder. We take the semiclassical approach, in which the effect of scattering is to cause transitions at random intervals from one wavepacket centered at $\vec{p}$ with $\varepsilon_{\vec{p}}=\varepsilon_{F}$ to another centered at $\vec{p}^{\prime}$ with $\varepsilon_{\vec{p}^{\prime}}=\varepsilon_{F}$, where $\varepsilon_{F}$ is the Fermi energy. This corresponds to a random switching in the direction of the effective magnetic field that acts on the spin degree of freedom. This is the D'yakonovPerel' mechanism of spin relaxation.[10, 11] The measured quantity in the continuous-wave experiments carried out on 2DEGs is $T_{2}$, the transverse relaxation time, while pulsed experiments can also measure $T_{1}$, the longitudinal relaxation time. For our purposes, a density matrix approach is the natural one, since we will eventually want to perform an ensemble average over all possible scattering sequences. Since the physical model of spins in a random time-dependent magnetic field is the same as that for relaxation of nuclear spins in liquids, the Redfield technique may be used.

We outline the calculation only briefly, since the details are parallel to the discussion in standard texts. 22 The $2 \times 2$ density matrix $\rho$ allows us to compute the expectation values of the spin by $\left\langle\sigma_{i}\right\rangle=\operatorname{Tr}\left\{\sigma_{i} \rho\right\}$. For a single system described by the Hamiltonian $H$, we have the equation of motion $d \rho / d t=\frac{i}{\hbar}[\rho, H]$. In the case of

$$
H=H_{0}+H_{1}(t)
$$




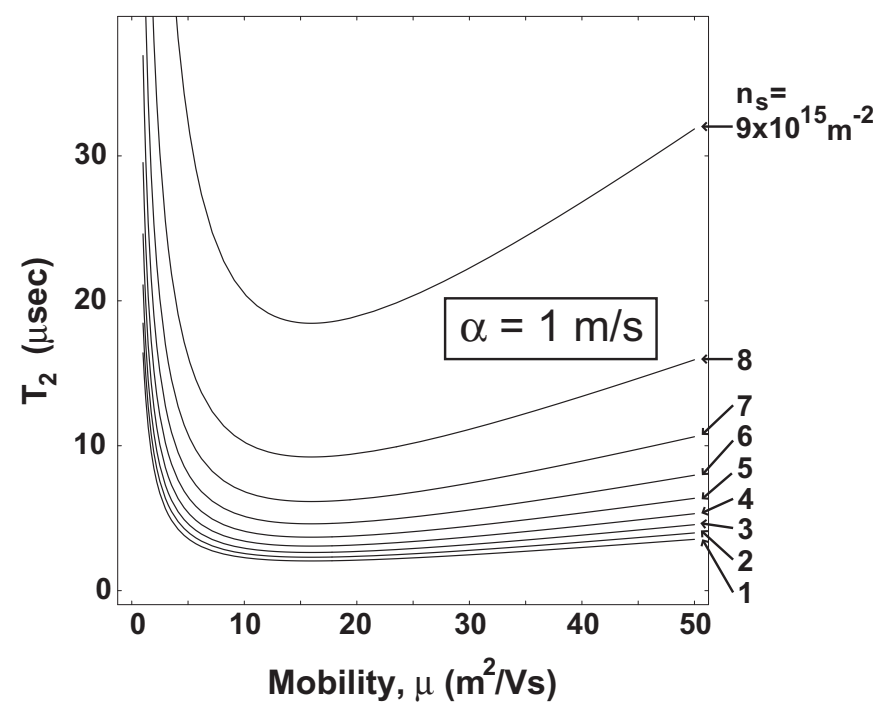

Figure 3: ESR linewidth lifetime $T_{2}$ from Eq. 16 for constant asymmetry coefficient, $\alpha=1 \mathrm{~m} / \mathrm{s}$, as a function of 2 DEG mobility, $\mu$, and density, $n_{s}$. For donor-layer populated quantum wells, divide the times listed by $\alpha^{2}: T_{2}(\alpha)=T_{2}(\alpha=1) / \alpha^{2}$. The magnetic field is assumed to be $B=0.33$ Tesla, perpendicular to the plane of the 2DEG.

where $H_{1}$ is small, it is convenient to go to the interaction representation

$$
\rho^{i n t}=\exp \left(-i H_{0} t / \hbar\right) \rho \exp \left(i H_{0} t / \hbar\right)
$$

and then we get

$$
\frac{d \rho^{i n t}}{d t}=\frac{i}{\hbar}\left[\rho^{i n t}, H_{1}^{i n t}(t)\right]
$$

where

$$
H_{1}^{i n t}(t)=\exp \left(i H_{0} t / \hbar\right) H_{1} \exp \left(-i H_{0} t / \hbar\right)
$$

This equation can be integrated to give

$$
\rho^{\text {int }}(t)=\rho^{\text {int }}(0)+\frac{i}{\hbar} \int_{0}^{t}\left[\rho^{\text {int }}\left(t^{\prime}\right), H_{1}^{i n t}\left(t^{\prime}\right)\right] d t^{\prime},
$$

and this can be solved interatively, which in second order gives

$$
\begin{aligned}
\frac{d \rho^{i n t}(t)}{d t} & =\frac{i}{\hbar}\left[\rho^{i n t}(0), H_{1}^{i n t}(t)\right] \\
& +\left(\frac{i}{\hbar}\right)^{2} \int_{0}^{t} d t^{\prime}\left[\left[\rho^{\text {int }}(0), H_{1}^{i n t}\left(t^{\prime}\right)\right], H_{1}^{i n t}(t)\right] .
\end{aligned}
$$

For example, let the steady field be in the $z$-direction, so that $H_{0}=\hbar \omega_{c} \sigma_{z} / 2$. The fluctuating field $\left[H_{1}^{i n t}(t)\right]_{s s^{\prime \prime}}=$ $\sum_{i=x, y} h^{i}(t) \sigma_{s s^{\prime \prime}}^{i}$ is in the transverse direction. The first order matrix element vanishes and we are left, in second order, with

$$
\begin{aligned}
\left(\frac{i}{\hbar}\right)^{-2}\left(\frac{d \rho_{s s^{\prime}}^{i n t}}{d t}\right)_{2}= & \sum_{s^{\prime \prime} s^{\prime \prime \prime}} \rho_{s s^{\prime \prime}}(0)\left[H_{1}^{i n t}\left(t^{\prime}\right)\right]_{s^{\prime \prime} s^{\prime \prime \prime}}\left[H_{1}^{i n t}(t)\right]_{s^{\prime \prime \prime} s^{\prime}} e^{i\left(s^{\prime \prime}-s^{\prime \prime \prime}\right) t^{\prime}} e^{i\left(s^{\prime \prime \prime}-s^{\prime}\right) t} \\
& +\sum_{s^{\prime \prime} s^{\prime \prime \prime}}\left[H_{1}^{i n t}(t)\right]_{s s^{\prime \prime}} e^{i\left(s-s^{\prime \prime}\right) t}\left[H_{1}^{i n t}(t)\right]_{s^{\prime \prime} s^{\prime \prime \prime}} e^{i\left(s^{\prime \prime}-s^{\prime \prime \prime}\right) t^{\prime}} \rho_{s^{\prime \prime \prime} s^{\prime}}(0) \\
& -\sum_{s^{\prime \prime} s^{\prime \prime \prime}}\left[H_{1}^{i n t}\left(t^{\prime}\right)\right]_{s s^{\prime \prime}} e^{i\left(s-s^{\prime \prime}\right) t^{\prime}} \rho_{s^{\prime \prime} s^{\prime \prime \prime}}^{i n t}(0)\left[H_{1}^{i n t}(t)\right]_{s^{\prime \prime \prime} s^{\prime}} e^{i\left(s^{\prime \prime \prime}-s^{\prime}\right) t} \\
& -\sum_{s^{\prime \prime} s^{\prime \prime \prime}}\left[H_{1}^{i n t}(t)\right]_{s s^{\prime \prime}} e^{i\left(s-s^{\prime \prime}\right) t} \rho_{s^{\prime \prime} s^{\prime \prime \prime}}^{i n t}(0) e^{i\left(s^{\prime \prime \prime}-s^{\prime}\right) t^{\prime}}\left[H_{1}^{i n t}\left(t^{\prime}\right)\right]_{s^{\prime \prime \prime} s^{\prime}},
\end{aligned}
$$


where we define $e^{s t}=e^{\epsilon_{s} t}$. Averaging the random field, we find

$$
\begin{aligned}
\overline{\left[H_{1}^{i n t}(t)\right]_{s s^{\prime \prime}}\left[H_{1}^{i n t}\left(t^{\prime}\right)\right]_{s^{\prime \prime} s^{\prime \prime \prime}}} & =\sum_{i j} \overline{h^{i}(t) h^{j}\left(t^{\prime}\right)} \sigma_{s s^{\prime \prime}}^{i} \sigma_{s^{\prime \prime} s^{\prime \prime \prime}}^{j} \\
& =\sum_{i=x, y, z} \chi^{i}\left(t-t^{\prime}\right) \sigma_{s s^{\prime \prime}}^{i} \sigma_{s^{\prime \prime} s^{\prime \prime \prime}}^{i}
\end{aligned}
$$

where $\tau=t-t^{\prime}$ and we define $\chi^{i}(\tau)=\overline{h^{i}(t) h^{i}\left(t^{\prime}\right)}$. We substitute this expression into Eq. 13 and do the matrix algebra. Eq. 13 can then be integrated in the limit where $t$ is large. We neglect the oscillating terms, which then yield

$$
\left(\frac{i}{\hbar}\right)^{-2}\left\langle\frac{d \rho_{++}}{d t}\right\rangle=\left[\chi^{x}\left(\omega_{c}\right)+\chi^{y}\left(\omega_{c}\right)\right]\left[\rho_{++}(0)-\rho_{--}(0)\right]
$$

and

$$
\left(\frac{i}{\hbar}\right)^{-2}\left\langle\frac{d \rho_{+-}}{d t}\right\rangle=\rho_{+-}(0)\left[\chi^{x}\left(\omega_{c}\right)+\chi^{y}\left(\omega_{c}\right)+2 \chi^{z}(0)\right]
$$

To obtain the relaxation times we must consider the equation for the spin:

$$
\begin{aligned}
\frac{d\left\langle\sigma^{z}\right\rangle}{d t} & =\frac{d\left[\operatorname{Tr}\left(\sigma^{z} \rho\right)\right]}{d t}=\frac{d}{d t}\left(\rho_{++}-\rho_{--}\right) \\
& =2\left(\frac{i}{\hbar}\right)^{2}\left[\chi^{x}\left(\omega_{c}\right)+\chi^{y}\left(\omega_{c}\right)\right]\left[\rho_{++}(0)-\rho_{--}(0)\right] \\
& =-2\left(\frac{1}{\hbar}\right)^{2}\left[\chi^{x}\left(\omega_{c}\right)+\chi^{y}\left(\omega_{c}\right)\right]\left\langle\sigma_{z}\right\rangle
\end{aligned}
$$

and so

$$
1 / T_{1}=2\left[\chi^{x}\left(\omega_{c}\right)+\chi^{y}\left(\omega_{c}\right)\right] / \hbar^{2} .
$$

Also

$$
\begin{aligned}
\frac{d\left\langle\sigma^{x}\right\rangle}{d t} & =\frac{d \operatorname{Tr}\left(\sigma^{x} \rho\right)}{d t}=\frac{d}{d t}\left(\rho_{+-}+\rho_{-+}\right) \\
& =-\left[\rho_{+-}(0)+\rho_{+-}(0)\right]\left[\chi^{x}\left(\omega_{c}\right)+\chi^{y}\left(\omega_{c}\right)+2 \chi^{z}(0)\right] / \hbar^{2} \\
& =-\left[\chi^{x}\left(\omega_{c}\right)+\chi^{y}\left(\omega_{c}\right)+2 \chi^{z}(0)\right]\left\langle\sigma_{x}\right\rangle / \hbar^{2}
\end{aligned}
$$

which gives

$$
1 / T_{2}=\left[\chi^{x}\left(\omega_{c}\right)+\chi^{y}\left(\omega_{c}\right)+2 \chi^{z}(0)\right] / \hbar^{2} .
$$

This gives a relation

$$
1 / T_{2}=1 / 2 T_{1}+2 \chi^{z}(0) / \hbar^{2} .
$$

We now wish to specialize to the case of a DP mechanism in a 2DEG. The main point is that the static field may be in any direction, while the fluctuationg field is in the plane. Consider first the special case that the static field is along $\widehat{z}$. Then

$$
1 / T_{2}=1 / 2 T_{1}=\left[\chi^{x}\left(\omega_{c}\right)+\chi^{y}\left(\omega_{c}\right)\right] / \hbar^{2} .
$$

Now consider a general direction, say $\vec{B}$ along the direction $B_{x} \widehat{x}+B_{z} \widehat{z}=\sin \theta \widehat{x}+\cos \theta \widehat{z}$, so that $\theta$ is the angle to the normal. Then the longitudinal fluctuations $\chi^{\|}$, which are quadratic in the field, are proportional to $\sin ^{2} \theta$ and the transverse ones $\chi^{\perp}$ to $\cos ^{2} \theta$. Thus

$$
1 / T_{1}(\theta)=2\left[\cos ^{2} \theta \chi^{x}\left(\omega_{L}\right)+\chi^{y}\left(\omega_{L}\right)\right] / \hbar^{2}
$$


while

$$
1 / T_{2}(\theta)=\left[\cos ^{2} \theta \chi^{x}\left(\omega_{L}\right)+\chi^{y}\left(\omega_{L}\right)+2 \sin ^{2} \theta \chi^{x}(0)\right] / \hbar^{2} .
$$

For the DP mechanism in a 2DEG the random field is constant in magnitude, but random in direction. The statistics of this field are Poisson: namely that if the $x$-component the field at time $t=0$ is $h^{x}$, then the chance of it remaining at $h^{x}$ decays as $\exp \left(-t / \tau_{p}\right)$. Hence

$$
\overline{h^{i}(0) h^{i}\left(\tau_{p}\right)}=\left\langle h_{x}^{2}\right\rangle e^{-t / \tau}=\frac{1}{2} \alpha^{2} p_{F}^{2} e^{-t / \tau_{p}}
$$

and

$$
\chi^{x, y}(\omega)=\frac{\alpha^{2} p_{F}^{2} \tau_{p}^{2}}{1+\omega^{2} \tau_{p}^{2}}
$$

In these formulas, $\tau_{p}$ is the momentum relaxation time. Note that these formulas assume s-wave scattering.

The zero-frequency limit of these formulas agrees with the recent results of Burkov and MacDonald 23] [see their Eq.(17)] . They do not agree with the formulas in Wilamowski et. al. 19], [see, e.g., their Eq.(3)] who state that the relaxation from the DP mechanism should vanish when $\theta=0$. This is not consistent with our results.

The DP mechanism has the nice feature that it is relatively easy to isolate experimentally. It is strongly anisotropic in the direction of the applied field compared to other mechanisms. To illustrate this we plot the ESR linewidths as a function of field angle in Fig. 2. What is most striking is the opposite dependence on angle for the rates $1 / T_{1}$ and $1 / T_{2}$, with $1 / T_{2}$ maximized when the field is in the plane of the $2 \mathrm{DEG}$, while $1 / T_{1}$ is maximized when the field is perpendicular to the plane of the 2DEG. Physically, this comes from the fact that the electric field is perpendicular, so that the fluctuations of the effective magnetic field are in the plane. Longitudinal relaxation $\left(T_{1}\right)$ is due to fluctuations perpendicular to the steady field, while transverse relaxation $\left(T_{2}\right)$ is due to fluctuations both perpendicular and parallel to the steady field. This mechanism has the characteristic that the change in $1 / T_{1}$ as the field is rotated through 90 degrees is always a factor of two. The change in $1 / T_{2}$ is frequency- and lifetime-dependent, with the anisotropy increasing as the mobility increases.

The DP relaxation also has the counter-intuitive inverse dependence of the spin relaxation time on the momentum relaxation time: $1 / T_{1,2} \propto \tau_{p}$, for small $\tau_{p}$ (or zero field), typical for motional narrowing. We plot the dependence of $T_{2}$ on the mobility in Fig. 3. At high mobilities and high frequencies $\omega>>1 / \tau_{p}$, we find $1 / T_{1,2} \propto 1 / \tau_{p}$.

\section{DISCUSSION}

We have calculated the transverse and longitudinal relaxation times of a silicon 2DEG in an arbitrary static magnetic field. To test our calculations, we compare them to known ESR data.[1, 2, 19, 24, 25] We limit ourselves to low temperatures, $\epsilon_{F} \sim 10-15 \mathrm{~K}$, and realistic material parameters for state-of-the-art heterostructures.

The most robust prediction of the theory is the anisotropy, particularly that of $T_{1}$, which is completely independent of all parameters. The only measurement, in Ref. [3], gives satisfactory agreement for $T_{1}: T_{1}^{B \| z} / T_{1}^{B \perp z}=0.67$ as opposed to the prediction 0.5 . Furthermore, the anisotropy of $1 / T_{2}$ goes in the opposite direction, as it should. The magnitude of this anisotropy is measured to be $T_{2}^{B \| z} / T_{2}^{B \perp z}=12.5$ which is about a factor of six larger than the theory predicts for the quoted mobility. The relaxation times, as far as can be determined by the range set by the uncertainty in silicon band parameters, are in rough agreement with what one gets from estimates assuming that this well is a device of type 1. The anisotropy of the 2DEG ESR linewidth is independent of $\alpha$ and is indeed only dependent on one free variable: the momentum relaxation time $\tau_{p}$ which we assume is directly proportional to the mobility. The magnitude of the relaxation time, on the other hand, is set by the Rashba coefficient together with the Fermi momentum.

Table【 details results for four other samples as well, also nominally of type 1 for which measurements of $T_{2}$ have been performed. Agreement is good for the sample of Ref. [19], particularly for the anisotropy of $T_{2}$. This is a donor-layer-populated sample measured with CW-ESR. Ref. [25], measured via electrically-detected ESR (ED-ESR), also is in good agreement with the anisotropy predicted by the theory. Comparison with the sample of Ref. 24] also seems to be very good. This is an IBM 2DEG with a density of roughly $4 \times 10^{15} \mathrm{~m}^{-2}$ and a quantum well thickness of $10 \mathrm{~nm}$, fully donor-layer populated. Agreement is considerably less good for the sample in Ref. [1]. Here the mobility is not well-known, and the sample is partially populated by illumination. So we are lead to believe that there is some difference between the two sets of samples that causes one set to have a larger $T_{2}$ anisotropy than our theory predicts, even for very similar material paramters (density and mobility). Further experimental work along the lines of measuring the density, mobility, $T_{1}$, and $T_{2}$ on the same samples is needed. 


\begin{tabular}{|c|c|c|}
\hline Source & Linewidth & Anisotropy \\
\hline $\begin{array}{c}\text { Ref. [19] } \\
5-30 \mathrm{~K} \\
\mu \sim 20 \mathrm{~m}^{2} / \mathrm{V}-\mathrm{s} \\
n_{s} \sim 4 \times 10^{15} \mathrm{~m}^{-2} \\
\text { CW-ESR } \\
\text { donor populated }\end{array}$ & $\begin{array}{c}\text { Exp.: } \\
T_{2}^{B \| z}=420 \mathrm{~ns}(0.15 \mathrm{G}) \\
T_{2}^{B \perp z}=140 \mathrm{~ns}(0.45 \mathrm{G}) \\
\text { Pred.: } \\
T_{2}^{B \| z}=191 \mathrm{~ns} \\
T_{2}^{B \perp z}=60 \mathrm{~ns}\end{array}$ & $\begin{array}{c}T_{2}^{B \| z} / T_{2}^{B \perp z}=3 \\
\\
T_{2}^{B \| z} / T_{2}^{B \perp z}=3.2 \\
T_{1}^{B \| z} / T_{1}^{B \perp z}=0.5\end{array}$ \\
\hline $\begin{array}{c}\text { Ref. [2] } \\
5 \mathrm{~K} \\
\mu \sim 9 \mathrm{~m}^{2} / \mathrm{V}-\mathrm{s} \\
n_{s} \sim 3 \times 10^{15} \mathrm{~m}^{-2} \\
\text { Pulsed-ESR } \\
\text { light populated }\end{array}$ & $\begin{array}{c}\text { Exp.: } \\
T_{2}^{B \| z}=3 \mu \mathrm{s} \\
T_{2}^{B \perp z}=0.24 \mu \mathrm{s} \\
T_{1}^{B \| z}=2 \mu \mathrm{s} \\
T_{1}^{B \perp z}=3 \mu \mathrm{S} \\
\text { Pred.: } \\
T_{2}^{B \| z}=502 \mathrm{~ns} \\
T_{2}^{B \perp z}=272 \mathrm{~ns} \\
T_{1}^{B \| z}=251 \mathrm{~ns} \\
T_{1}^{B \perp z}=502 \mathrm{~ns}\end{array}$ & $\begin{array}{c}T_{2}^{B \| z} / T_{2}^{B \perp z}=12.5 \\
T_{1}^{B \| z} / T_{1}^{B \perp z}=0.67 \\
T_{2}^{B \| z} / T_{2}^{B \perp z}=1.8 \\
T_{1}^{B \| z} / T_{1}^{B \perp z}=0.5\end{array}$ \\
\hline $\begin{array}{c}\text { Ref. [1] } \\
\mu \sim 10 \mathrm{~m}^{2} / \mathrm{V}-\mathrm{s} \\
n_{s} \sim 3 \times 10^{15} \mathrm{~m}^{-2} \\
\text { CW-ESR } \\
\text { light/gate populated }\end{array}$ & $\begin{array}{c}\text { Exp.: } \\
T_{2}^{B \| z}=12 \mu \mathrm{s} \\
T_{2}^{B \perp z}=500 \mathrm{~ns} \\
\text { Pred.: } \\
T_{2}^{B \| z}=480 \mathrm{~ns} \\
T_{2}^{B \perp z}=193 \mathrm{~ns}\end{array}$ & $\begin{array}{l}T_{2}^{B \| z} / T_{2}^{B \perp z}=24 \\
T_{2}^{B \| z} / T_{2}^{B \perp z}=1.9 \\
T_{1}^{B \| z} / T_{1}^{B \perp z}=0.5\end{array}$ \\
\hline $\begin{array}{c}\mu \sim 5 \mathrm{~m}^{2} / \mathrm{V}-\mathrm{s} \\
n_{s} \sim 4 \times 10^{15} \mathrm{~m}^{-2} \\
\mathrm{CW}-\mathrm{ESR} \\
\text { donor populated }\end{array}$ & $\begin{array}{c}\text { Exp.: } \\
T_{2}^{B \| z}=50 \mathrm{~ns}(0.13 \mathrm{G}) \\
T_{2}^{B \perp z}=30 \mathrm{~ns}(0.215 \mathrm{G}) \\
\text { Pred.: } \\
T_{2}^{B \| z}=31 \mathrm{~ns} \\
T_{2}^{B \perp z}=20 \mathrm{~ns}\end{array}$ & $\begin{array}{l}T_{2}^{B \| z} / T_{2}^{B \perp z}=1.65 \\
T_{2}^{B \| z} / T_{2}^{B \perp z}=1.6 \\
T_{1}^{B \| z} / T_{1}^{B \perp z}=0.5\end{array}$ \\
\hline $\begin{array}{c}\text { Ref. [25] } \\
4.2 \mathrm{~K} \\
\mu \sim 9 \mathrm{~m}^{2} / \mathrm{V}-\mathrm{s} \\
n_{s} \sim 4 \times 10^{15} \mathrm{~m}^{-2} \\
\text { ED-ESR } \\
\text { donor populated }\end{array}$ & $\begin{array}{c}\text { Exp.: } \\
T_{2}^{B \| z}=105 \mathrm{~ns}(0.6 \mathrm{G}) \\
T_{2}^{B \perp z}=49 \mathrm{~ns}(1.3 \mathrm{G}) \\
\text { Pred.: } \\
T_{2}^{B \| z}=212 \mathrm{~ns} \\
T_{2}^{B \perp z}=115 \mathrm{~ns}\end{array}$ & $\begin{array}{l}T_{2}^{B \| z} / T_{2}^{B \perp z}=2.2 \\
T_{2}^{B \| z} / T_{2}^{B \perp z}=1.8 \\
T_{1}^{B \| z} / T_{1}^{B \perp z}=0.5\end{array}$ \\
\hline
\end{tabular}

Table I: We calculate relaxation times assuming that the QW is completely populated by the donor layer. Accurate analysis is made difficult due to lack of precise values for mobility and density, which are often not measured directly (or reported) for the specific sample addressed with ESR. The anisotropy does not depend on the Rashba coefficient. Note also that there is some disagreement in the literature as to how to convert from linewidth to a relaxation time, we use the equations derived by Poole in Ref. [26], $T_{2}=2 \hbar /\left(\sqrt{3} g \mu_{B} \Delta H_{p p}^{0}\right)$, but others may differ by a factor of up to $2 \pi$.

The magnitude of the predicted relaxation times is in general well predicted by the theory, but there is a range of error. The position of the ionized centers which populate the well is important for the calculation of the electric field which is thus hard to characterize. Photoelectrons created with light at the bandgap energy may also have symmetry changing effects. This may explain why the Rashba coefficient derived from varying the 2DEG density by light in Ref. [19] appears to be independent of density. It is also important to point out that parallel conductivity (current paths through both the 2DEG and the donor-layer for example) is a common problem in today's SiGe quantum wells and may effect the transport measurements of density and especially mobility making comparison with theory difficult.

Other mechanisms may become important as we leave the parameter range considered in this paper. Electronelectron collisions which do not greatly affect the mobility at low temperatures may start to contribute at higher temperatures and mobilities, as they appear to do in GaAs quantum wells. 227] These collisions will also relax the spin, but the relation between momentum relaxation and spin relaxation is not expected to be the same as for the 
elastic collisions considered here. At higher magnetic fields, the cyclotron motion of the electrons is important. In the semiclassical picture, when $\omega_{c} \tau_{p} \geq 1$, the average value of the momentum perpendicular to the magnetic field shrinks, reducing or even eliminating DP spin relaxation, as has been considered for III-V semiconductors in the fixed magnetic field case.[12, 28] This effect may become important at high mobilities and would be dependent on magnetic field angle, increasing the anisotropy predicted here while also increasing the relaxation times. Quantum effects may become important in this regime however. The wave vector dependence of the conduction band electron g-factor may also lead to relaxation, as has been pointed out recently, [29] but hasn't been considered here. Finally, the addition of details related to the presence of two conduction-band valleys may differentiate further the case of $\mathrm{Si}$ from that of GaAs. Golub and Ivchenko [30] have considered spin relaxation in symmetrical $(\alpha=0)$ SiGe QWs, where valley domains (even or odd monolayer regions of the QW) may have influence over spin dynamics. Random spinorbit coupling due to variations in the donor layer charge distribution may also be important in symmetric quantum wells. 31, 32]

Long spin relaxation times on the order of hundreds of nanoseconds to microseconds, found in presently available SiGe quantum wells, hold great promise for both quantum information processing and spintronics. Our results demonstrate that decreasing the reflection asymmetry within the device will appreciably decrease the Rashba coefficient and the consequent spin relaxation at low temperatures. This can be achieved by a symmetric doping profile or using an external electric field to cancel out the field of the ions. They further show that the anisotropy of the ESR linewidth as a function of angle may be a good indicator of 2DEG quality (mobility) independent of transport measurements. As higher mobility and more exotic SiGe heterostructures are grown and characterized, new physics may emerge.

\section{Acknowledgements}

The authors would like to acknowledge useful conversations with Mark Friesen and Jim Truitt and with the rest of the Wisconsin-Madison solid-state quantum computing group.[34] This work has been supported by the Army Research Office through ARDA and the NSF QUBIC program NSF-ITR-0130400.

[1] W. Jantsch, Z. Wilamowski, N. Sanderfeld, M. Muhlberger, and F. Schaffler, Physica E 13, 504 (2002).

[2] A. M. Tyryshkin, S. A. Lyon, A. V. Astashkin, and A. M. Raitsimring, arxiv/cond-mat 0304284 (2003).

[3] A. M. Tyryshkin, S. A. Lyon, A. V. Astashkin, and A. M. Raitsimring, Phys. Rev. B 68, 193207 (2003).

[4] R. de Sousa and S. D. Sarma, Phys. Rev. B 68, 115322 (2003).

[5] L. Roth, Phys. Rev. 118, 1534 (1960).

[6] H. Hasegawa, Phys. Rev. 118, 1523 (1960).

[7] A. V. Khaetskii and Y. V. Nazarov, Phys. Rev. B 64, 125316 (2001).

[8] D. Pines, J. Bardeen, and C. P. Slichter, Phys. Rev. 106, 489 (1957)

[9] D. K. Wilson and G. Feher, Phys. Rev. 124, 1068 (1961).

[10] M. D'yakonov and V. Perel', Sov. Phys. Solid State 13, 3023 (1972).

[11] M. D'yakonov and V. Kachorovskii', Sov. Phys. Semicond. 20, 110 (1986).

[12] B. P. Zakharchenya, Modern Problems in CM Sciences V.8: Optical Orientation (North-Holland, 1984).

[13] M. Friesen, P. Rugheimer, D. E. Savage, M. G. Lagally, D. W. van der Weide, R. Joynt, and M. A. Eriksson, Phys. Rev. B 67, 121301 (2003).

[14] M. H. Jones and S. H. Jones, Virginia Semiconductor (2002).

[15] H. Jiang and E. Yablonovitch, Phys. Rev. B 64, 041307(R) (2001).

[16] Y. Bychkov and E. Rashba, J. Phys. C 17, 6039 (1984).

[17] E. A. de Andrada e Silva, G. C. L. Rocca, and F. Bassani, Phys. Rev. B 55, 16293 (1997).

[18] P. Lawaetz, Phys. Rev. B 4, 3460 (1971).

[19] Z. Wilamowski, W. Jantsch, H. Malissa, and U. Rossler, Phys. Rev. B 66, 195315 (2002).

[20] A. V. Khaetskii and Y. V. Nazarov, Phys. Rev. B 61, 12639 (2000).

[21] L. Cui, Y. Zeng, B. Wang, Z. Zhu, L. Lin, C. Jiang, S. Guo, and J. Chu, Appl. Phys. Let. 80, 3132 (2002).

[22] C. P. Slichter, Principles of Magnetic Resonance (Springer-Verlag, 1978).

[23] A. A. Burkov and A. H. MacDonald, arxiv/cond-mat 0311328 (2003).

[24] J. Truitt (2004).

[25] C. Graeff, M. Brandt, M. Stutzmann, M. Holzmann, G. Abstreiter, and F. Schaffler, Phys. Rev. B 59, 13242 (1999).

[26] C. P. Poole, Electron Spin Resonance (Dover Publications Inc., 1983), 2nd ed.

[27] M. Glazov, E. Ivchenko, M. Brand, O. Karimov, and R. Harley, arxiv/cond-mat 0305260 (2003).

[28] E. Ivchenko, Sov. Phys. Solid State 15, 1048 (1973).

[29] F. X. Bronold, I. Martin, A. Saxena, and D. L. Smith, Phys. Rev. B 66, 233206 (2002).

[30] L. Golub and E. Ivchenko, Phys. Rev. B 69, 115333 (2004). 
[31] E. Y. Sherman, Appl. Phys. Let. 82, 209 (2003).

[32] E. Y. Sherman, Phys. Rev. B 67, 161303(R) (2003).

[33] Note that [19] uses the wrong expression for the Fermi wavevector, failing to include the valley degeneracy, so their data analysis is off by a factor of sqrt(2).

[34] http://qc.physics.wisc.edu/ 